\title{
敦-
}

\section{Perfil socio-demográfico de madres adolescentes en España}

\author{
Ana Lucía Hernández Cordero \\ Departamento de Psicología y Sociología \\ UNIVERSIDAD DE ZARAGOZA \\ acordero@unizar.es \\ Alessandro Gentile \\ Departamento de Psicología y Sociología \\ UNIVERSIDAD DE ZARAGOZA \\ agentile@unizar.es \\ ELENA LUMINITA TANASE \\ Departamento de Psicología y Sociología \\ UNIVERSIDAD DE ZARAGOZA \\ elenaluminita19@gmail.com
}

Resumen: En el artículo describimos cómo han evolucionado la maternidad adolescente y los embarazos tempranos en España desde mediados de los años noventa hasta 2017. Gracias a la explotación de datos secundarios de encuestas nacionales y al manejo de los hallazgos 
de numerosas investigaciones anteriores a la nuestra, destacamos las conductas afectivo-sexuales de las adolescentes residentes en este país y nos detenemos en el perfil socio-demográfico de aquellas chicas que han dado a luz antes de haber cumplido los 20 años durante este periodo.

Palabras claves: conductas de riesgo, embarazos tempranos, maternidad adolescente, tasa de fecundidad adolescente, transición a la vida adulta.

\section{Socio-demographic profile of teenage mothers in Spain}

Abstract: In this paper we describe the evolution of teenage maternity and early pregnancies in Spain from the mid-1990s until 2017. Thanks to the examination of secondary data from national surveys and the handling of findings from a large body of research carried out prior to our own, we draw attention to the sexual and affective behaviours of adolescents residing in this country and take a closer look at the socio-demographic profile of those girls that have given birth before the age of 20 during this period.

Key Words: risk behaviours, teenage pregnancies, teenage maternity, teenage fertility rate, transition to adulthood. 


\section{Perfil socio-demográfico de madres adolescentes}

en España

Ana Lucía Hernández Cordero

Alessandro

Gentile

https://doi.org/10.26754/ojs_ais/ais.2019404199

Recibido: 22/05/2019

Aceptado: 15/10/2019

Elena

Luminita Tanase

El artículo se adscribe a la investigación "Relatos de Madres adolescentes en la España actual" patrocinado por el Centro Reina Sofía sobre Adolescencia y Juventud de Madrid, por la Dirección General de Igualdad y Familias del Gobierno de Aragón y por el Grupo de Estudio sobre la Sociedad del Riesgo de la Universidad de Zaragoza.

\section{INTRODUCCIÓN}

En España la maternidad y los embarazos tempranos ${ }^{1}$ han sido poco estudiados en sus causas y efectos sociales hasta principios de los años noventa, cuando se empieza a recopilar datos sobre cuántas y quiénes son las jóvenes madres. Algunos de los fenómenos sociales que explican los riesgos de embarazos no deseados al que se exponen las adolescentes españolas en las últimas décadas son la importancia de la sexualidad para nuestros jóvenes (Ayuso y García, 2014), el creciente acceso a métodos anticonceptivos y el aumento de la actividad sexual desvinculada de la finalidad reproductiva (Alberdi, 2014), de las interrupciones voluntarias del embarazo (IVE) en las menores de edad y de las violencias de

${ }^{1}$ Los embarazos se consideran precoces (antes de los 14 años de edad) o tempranos (entre 15 y 19 años) en función de la madurez biológica de la mujer para llevar la gestación en condiciones de bajo riesgo (OMS, 2014). 
género que se manifiestan con cada vez mayor frecuencia en los centros escolares (Díaz-Aguado, 2006).

En el artículo sistematizamos datos procedentes de encuestas nacionales como la II Encuesta sobre Sexualidad y Juventud Española (Grupo Daphne, 2009), la VII Encuesta de Anticoncepción en España (Grupo Daphne, 2011) y las Encuestas de Anticoncepción 2014 y 2018 de la Sociedad Española de Contracepción; así como de investigaciones recientes sobre el tema haciendo hincapié en tres aspectos: 1) las conductas afectivos-sexuales de los/las jóvenes; 2) los nacimientos en las adolescentes entre 1996 y 2017; 3) los rasgos socio-demográficos más destacados de estas madres en el mismo periodo. El resultado de estas operaciones es un análisis de la maternidad adolescente a lo largo de los últimos veinte años en España, demostrando que se trata de un fenómeno poco visible en nuestra sociedad, pero actual y en evolución. Abordar esta temática se convierte en una oportunidad para reflexionar sobre los embarazos no deseados entre adolescentes, sobre las estructuras de oportunidades existentes para los jóvenes para prevenirlos y sobre las circunstancias sociales, culturales e institucionales que los propician. Tales reflexiones son imprescindibles para plantear actuaciones no solo preventivas, sino también acertadas, duraderas y no estigmatizadoras.

\section{CONDUCTAS AFECTIVO-SEXUALES DE JÓVENES Y ADOLESCENTES}

Entre los factores que mejor explican los embarazos en edad adolescente cabe mencionar la importancia de la sexualidad en el proceso de transición a la vida adulta, como conducta que implica la búsqueda del placer y el desarrollo de lazos afectivos, junto con el aumento de las prácticas anticonceptivas y del acceso legal a las IVE. Según el estudio Jóvenes y diversidad sexual (INJUVE, 2010) sobre una muestra nacional de jóvenes de 15-29 años el $73,2 \%$ de ellos está bastante o muy de acuerdo en afirmar que la sexualidad ocupa un lugar desta cado en su vida. Esta valoración aumenta con la edad: el sexo es una práctica relevante en las relaciones de pareja (esporádicas o estables) para la mayoría de chicos y chicas de $15-19$ años $(53,6 \%)$ y esencial para los veinteañeros $(75,7 \%$ en la cohorte de $20-24$ años y $83,9 \%$ para los de 25-29 años). 
Gracias a la explotación de la Encuesta de Fecundidad y Valores 2006 del Centro de Investigaciones Sociológicas, un estudio dirigido por Delgado (2011) muestra que el $20 \%$ de las mujeres nacidas antes de 1956 tienen su primera relación sexual completa cuando aún no han cumplido los 20 años; este porcentaje llega al $52,7 \%$ para las nacidas entre 1956 y 1970 y aumenta al $74 \%$ en la cohorte 1971-1985.

La edad media de la primera relación sexual completa se adelanta 5,6 años desde los años sesenta. El número de mujeres que pierde su virginidad antes de los 16 años se multiplica por doce y en 2010 representa el 16,7\% de la población juvenil (Lailla, 2011). La III Encuesta sobre Sexualidad y Juventud Española (Grupo Daphne, 2009) evidencia un aumento de la población de 15-19 años sexualmente activa respecto al 2005, cuando se realizó la segunda relevación empírica de este tipo, con una subida mayor para las chicas (de 52,8\% en 2005 a 65,7\% en 2009) respecto a los chicos (de 55,8\% a 67,1\%). La Encuesta de Anticoncepción SEC 2018 fija la edad media del debut sexual para las jóvenes en 16,3 años.

Las generaciones recientes de mujeres adelantan sus relaciones sexuales desvinculándolas de la finalidad reproductiva gracias al uso de métodos anticonceptivos (Castro-Martín, 2007). Hasta 1978 la contracepción en España estaba prohibida por ley. Un médico podía prescribir la píldora (introducida en Europa en 1961) únicamente para regular el ciclo menstrual. Desde finales de los años setenta, tras su despenalización, la contracepción favorece la reducción de embarazos entre las jóvenes (Alberdi, 2014). La VII Encuesta de Anticoncepción en España (Grupo Daphne, 2011) señala que en 2004 el 46,6\% de las mujeres nacidas entre 1985 y 1990 emplea algún tipo de método anticonceptivo; en 2009 esta proporción aumenta al 62,6\% para las adolescentes del quinquenio sucesivo, 1990-1995.

Aunque el uso del preservativo ahora sea mucho más extendido entre la juventud española (con una media del $74,4 \%$ en 2010 según el Informe de Juventud en España 2012) hasta 2004 el $17 \%$ de los chicos y el $10 \%$ de las chicas de $15-24$ años empleaban el coitus interruptus como medida de anticoncepción, y un $6 \%$ declaraba haber acudido algunas veces a la "píldora del día después" como recurso de emergencia (Megías et al., 2005). 
La edad media a partir de la cual las españolas comienzan a utilizar de forma sistemática algún método de anticoncepción (excepto el coitus interruptus) es de 20,7 años (SEC, 2014), cuando la frecuencia de los emparejamientos sexuales se intensifica. Sin embargo, el uso de algún tipo de protección en la primera relación sexual ha aumentado en las menores de 20 años, según la Encuesta de Anticoncepción SEC 2018 el 97,3\% de las jóvenes que pertenecen a este grupo de edad ha utilizado algún tipo de anticoncepción.

Según la III Encuesta sobre Sexualidad y Juventud Española (Grupo Daphne, 2009) dos de cada tres jóvenes de la cohorte de 15-19 años mantienen relaciones sexuales con una frecuencia de 6,9 veces al mes (ellos) y 8,7 (ellas). Mientras que para los de 2024 años la frecuencia aumenta llegando a 8,8 y 9,5 veces al mes para chicos y chicas (INJUVE, 2008). Según la misma encuesta del Grupo Daphne (2009) el 13,2\% de los jóvenes no se ha cuidado con ningún método anticonceptivo en el último encuentro sexual y sólo el $66 \%$ lo utiliza de forma habitual. Sin embargo, más del $94 \%$ de los jóvenes con una vida sexual activa declaran saber cómo se maneja eficazmente por lo menos un método anticonceptivo.

Aunque tengan cada vez más acceso a los recursos de anticoncepción, no todos los jóvenes saben utilizarlos correctamente: el último estudio Daphne (2011) informa que el 23,1\% de los adolescentes de 15-19 años que se protegen no lo hace de forma adecuada. Esta cuestión se puede explicar por una educación sexual incompleta o mal orientada en los centros escolares y en las familias o porque ellos mismos relativizan el verdadero impacto de sus acciones y se dejan llevar por "falsos mitos" referidos al uso de anticonceptivos (Madrid, 2009; Yago y Tomás, 2014). Según la III Encuesta sobre Sexualidad y Juventud Española del Grupo Daphne 387.000 jóvenes de 15-24 años en 2009 se exponen a embarazos no deseados por un mal uso del preservativo. Entre los elementos que influyen en la ocurrencia de estos embarazos y de las Enfermedades de Transmisión Sexual (ETS) para los adolescentes en España destacamos: la escasa presencia en el territorio nacional de unos servicios sanitarios especializados donde los menores puedan acudir sin sus padres, la carencia de valores éticos y morales de referencia en cuanto a una educación afectivo-sexual integral basada en el respeto, la influencia 
excesiva de información de unos medios de comunicación que promueven modelos desiguales de relaciones de género y el insuficiente control de parte de los adultos (familiares y no) (Lailla, 2011: 122).

\section{LA INTERRUPCIÓN VOLUNTARIA DEL EMBARAZO DE LAS JÓVENES}

Hasta 1985 abortar es un delito en España. La Ley Orgánica 9/1985 de reforma del Artículo 417bis del Código Penal establece un sistema de supuestos que solo permite realizar la IVE en casos de violación, malformación del feto o riesgo para la salud de la madre.

En 2010 se aprueba la Ley Orgánica 2/2010 de Salud Sexual y Reproductiva y de la Interrupción Voluntaria del Embarazo que deroga la anterior ley de supuestos y permite el aborto libre en las 14 primeras semanas. La norma contempla que se puede practicar un aborto también dentro de las 22 semanas de gestación, en el caso de que exista grave riesgo para la vida o la salud de la embarazada o de serias anomalías en el feto, y más allá de este plazo, en cualquier momento si se detectan alteraciones fetales incompatibles con la vida del niño o enfermedades incurables.

Con esa ley se establece que las chicas de 16 y 17 años pueden interrumpir su embarazo sin el consentimiento de los padres ${ }^{2}$. Este ha sido uno de los puntos más controvertidos en el debate social y político alrededor de la reforma de 2010, porque muchos temen que tales disposiciones pueden disparar el número de IVE entre las adolescentes. Sin embargo, un dato significativo que describe sus conductas reales de acuerdo con lo establecido por esta ley es lo que presenta la Asociación de Clínicas Acreditadas para la Interrupción del Embarazo, según la cual el 87,3\% de las chicas de 16 y 17 años que practican un IVE en 2011 son acompañadas al menos por uno de sus padres o por su tutor legal. Además, el número total de las IVE practicadas por las menores de 20 años bajan paulatinamente a partir de 2012 .

${ }^{2}$ El asunto es distinto para las menores de 16 años que deben informar al menos a uno de los padres o a sus representantes legales acerca de su decisión de abortar, excepto si se alegan pruebas de violencia intrafamiliar o si se encuentran en situaciones de desamparo, en cuyo caso la petición de IVE tiene que recogerse bajo un informe de los servicios sociales. 
Hoy en día sigue en vigor la Ley de plazos de 2010, pero se introduce una modificación con la Ley Orgánica 11/2015 estableciendo que todas las embarazadas menores de 18 años necesitan el consentimiento expreso de los titulares de la patria potestad (progenitores o representantes legales) para practicarse una IVE $^{3}$. Según el legislador el objetivo principal de esta medida es "reforzar la protección de las menores".

En este nuevo marco normativo, la tasa de abortos voluntarios $^{4}$ entre las chicas de 15-19 años pasa de 4,8 en 1996 (un total de 7.331) a 8,8 en 2017 (9.755 casos) tras haber alcanzado el máximo de 13,6 en 2007 (15.307).

\section{NACIMIENTOS DE MADRES ADOLESCENTES DESDE 1996 HASTA LA ACTUALIDAD}

Si la joven decide llevar a cabo el embarazo y/o si se da la circunstancia de que ya no puede optar por el IVE legalmente, el bebé que va a nacer será acogido por una madre adolescente. La tasa de fecundidad adolescente ${ }^{5}$ baja de forma pronunciada desde los años ochenta, estabilizándose en la década de los noventa y registra un repunte a principios del nuevo siglo: el número de los nacimientos de madres adolescentes crece entre 1996 (11.174) y 2008 (15.133) con un cambio de tendencia en los años sucesivos, reduciéndose casi un 50\% en 2017 (7.839). Esto significa que la ratio sobre el total de estos tipos de nacimientos ocurridos en España pasa de 3,08\% a 1,99\% entre 1996 y 2017 (Tabla 1).

${ }^{3} \mathrm{Si}$ hay conflicto o desacuerdo entre la menor y sus padres sobre el consentimiento para que ella pueda abortar, éste se resolverá judicialmente según determina el Código Civil Español.

${ }^{4}$ Es decir, la proporción de IVE por 1.000 mujeres en edad fértil registrada anualmente por el Ministerio de Sanidad, Consumo y Bienestar Social

${ }^{5}$ Eso es, la relación entre el número de nacidos vivos y la cantidad de población femenina de 15-19 años expresada como el número de nacimientos por cada 1.000 de esas mujeres. 


\section{Tabla 1}

Indicadores de referencia para describir las proporciones de nacimientos de madres adolescentes (15-19 años) en España

\begin{tabular}{|c|c|c|c|c|c|}
\hline & $\begin{array}{c}\text { Número } \\
\text { nacimientos } \\
\text { de menores } \\
\text { de 20 años* }\end{array}$ & $\begin{array}{c}\text { Número } \\
\text { total de } \\
\text { nacimientos }\end{array}$ & $\begin{array}{c}\text { Ratio sobre } \\
\text { total } \\
\text { Nacimientos }\end{array}$ & $\begin{array}{c}\text { Mujeres } \\
\text { residentes } \\
\mathbf{1 5 - 1 9} \text { años }^{\star \star *}\end{array}$ & $\begin{array}{c}\text { Tasa de } \\
\text { fecundidad } \\
\text { adolescente** }^{\star *}\end{array}$ \\
\hline 1996 & $11.174(99)$ & 362.626 & $\mathbf{3 , 0 8}$ & 1.501 .814 & $(x / z)^{\star} 1000$ \\
\hline 2000 & $11.386(102)$ & 397.632 & $\mathbf{2 , 8 6}$ & 1.249 .614 & $\mathbf{7 , 3 7}$ \\
\hline 2004 & $12.496(140)$ & 454.591 & $\mathbf{2 , 7 4}$ & 1.133 .437 & $\mathbf{1 0 , 9 0}$ \\
\hline 2008 & $15.133(178)$ & 519.779 & $\mathbf{2 , 9 1}$ & 1.116 .716 & $\mathbf{1 3 , 3 9}$ \\
\hline 2012 & $9.724(143)$ & 454.648 & $\mathbf{2 , 1 3}$ & 1.051 .130 & $\mathbf{9 , 1 1}$ \\
\hline 2015 & $8.305(98)$ & 420.290 & $\mathbf{1 , 9 7}$ & 1.060 .191 & $\mathbf{7 , 7 4}$ \\
\hline 2017 & $7.839(114)$ & 393.181 & $\mathbf{1 , 9 9}$ & 1.096 .394 & $\mathbf{7 , 1 5}$ \\
\hline
\end{tabular}

* Entre paréntesis los nacimientos de madres menores de 15 años de edad

** Para esta operación se han sustraído el número de los nacimientos de menores de 15 años (entre paréntesis) del valor $x$ de referencia en cada año considerado

*** Se indica el número total de residentes en España al 1 de enero del año sucesivo respecto al año de referencia (estos datos proceden del censo demográfico del INE)

Fuente: Estadística de Nacimientos, Movimiento natural de la población (INE)

Si exceptuamos la tasa de fecundidad adolescente en 2008 , como indicador del aumento más grande de los últimos veinte años, observamos poca variación de la frecuencia de nacimientos de madres adolescentes entre 1996 y 2017; esto quiere decir que en 1996 de cada 1.000 adolescentes residentes en España 7,37 mujeres menores de 20 años se embarazan y dan a luz mientras que en 2017 estos acontecimientos se dan en 7,15 adolescentes por cada 1.000 residentes en el país. Estos datos llaman mucho la atención: aunque haya disminuido un $23 \%$ la población femenina de 15-19 años residente en España durante el mismo periodo (1.501.814 en 1996 frente a 1.096.394 de 2017) la frecuencia de adolescentes con hijos baja solamente 2 puntos decimales ${ }^{6}$.

${ }^{6}$ La tasa de natalidad de nuestras adolescentes en 2013 era 10,11 mientras que las proporciones más altas de nacimientos de madres precoces se registraron en España entre 1974 y 1983: durante este periodo la tasa de fecundidad ha estado siempre superior a 20 por cada 1.000 , con el punto más alto en $1977(25,6)$. 


\section{DISTRIBUCIÓN REGIONAL DE LOS NACIMIENTOS DE MADRES ADOLESCENTES}

La concentración de madres adolescentes en algunas Comunidades Autónomas más que en otras es un indicador de la brecha estructural existente entre los diversos territorios del estado (Delgado, 2011). Las regiones con mayores índices de estudios superiores, de renta per cápita y de participación femenina en el mercado de trabajo entre su población, tienen una tasa de fecundidad adolescente hasta un $40 \%$ menor respecto a las menos dinámicas en términos socio-económicos, según estos indicadores.

En 1996 la tasa de fecundidad adolescente más alta (superior a 10) sobre la población residente con menos de 20 años se registra en Canarias, Murcia, Andalucía y Extremadura, frente a los valores más bajos (inferior a 4) de la Comunidad de Madrid, Navarra, Aragón, La Rioja y País Vasco (Gráfico 3). Podríamos explicar esta variedad con base en unos diferentes patrones culturales de cada región que caracterizan la vida sexual y reproductiva de esas jóvenes, sus pautas de transición a la vida adulta y la creación de nuevos hogares. Tendríamos así unos modelos de referencia más conservadores en el sur, donde es mayor la presencia de población rural y de un grupo étnico muy tradicionalista como el gitano, con emancipación juvenil temprana orientada a la formación de una familia con hijos (San Román, 1997); y otros más secularizados en el norte, con más industrialización y una población más concentrada en áreas urbanas, con emancipación juvenil más tardía y caracterizada por un abanico más heterogéneo de opciones de vida independiente. Esta lectura del fenómeno está reforzada por el estudio de Delgado (2011) que en su relevación empírica de 2006 señala que en Andalucía, Extremadura y Murcia el $78 \%$ de las mujeres que han sido madres en la adolescencia llegan a tener un segundo hijo antes de los 25 años, frente al $56 \%$ en Madrid, Navarra y País Vasco (Gráfico 1). 


\section{Gráfico 1 \\ Tasa de fecundidad adolescente por Comunidad Autónoma (1996-2015)}

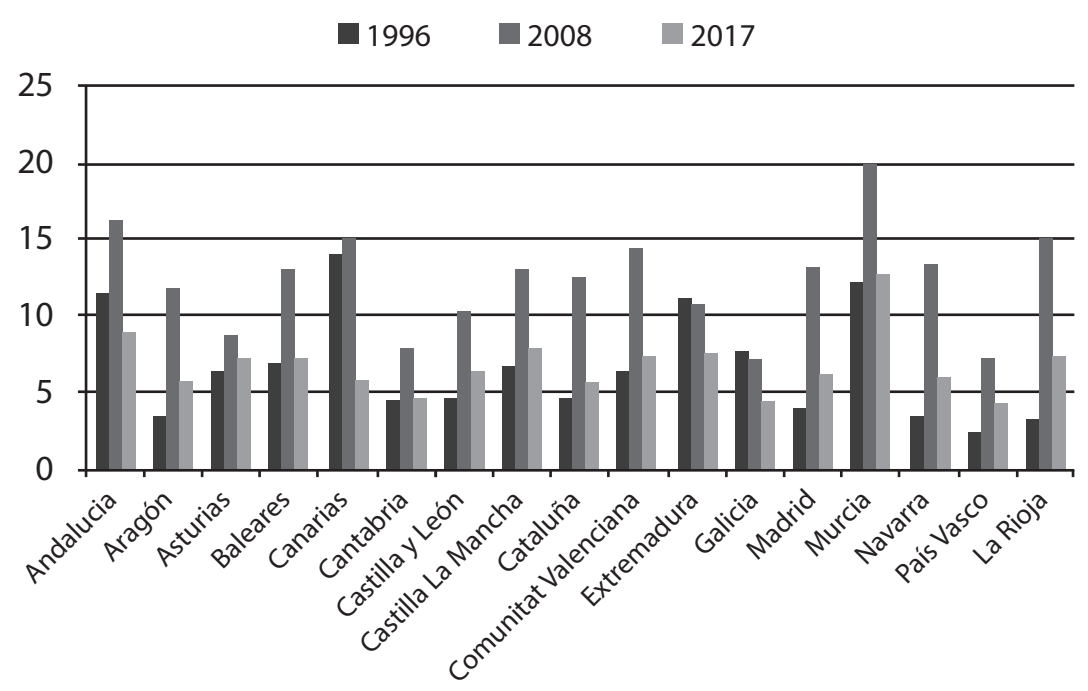

Fuente: Elaboración propia a partir de Estadística de nacimientos,. Movimiento natural de la población (INE)

La evolución de las tasas de fecundidad adolescente a nivel regional entre 1996 y 2017 impone una reflexión ulterior: exceptuando el estancamiento en la región de Murcia (que sigue teniendo una tasa de fecundidad adolescente muy alta, cercana a 12,3 hijos por cada 1.000 mujeres de 15-19 años allí residentes) el valor de este indicador baja en Andalucía (de 11,4 a 8,9) y Extremadura $(11,1$ a 7,6$)$ y se reduce de casi dos tercios en Canarias (de 14,1 a 5,8) mientras que aumenta sensiblemente en la Comunitat Valenciana (de 6,4 a 7,3), en Islas Baleares (de 6,8 a 7,2), en Asturias (de 6,4 a 7,1), en las dos Castillas (de 4,6 a 6,2 en Castilla-León y de 6,6 a 7,9 en Castilla-La Mancha), en Cataluña (de 4,7 a 5,6) y en Aragón (de 3,4 a 5,6), hasta casi doblarse en Madrid (de 3,9 a 6,2) y en las regiones del norte (como en el caso de Navarra, de 3,3 a 6,1, y País Vasco, de 2,3 a 4,3), registrando la mayor subida en La Rioja (de 3,2 a 7,2).

Aunque la tasa de fecundidad adolescente siga siendo comparativamente más alta en las regiones del sur, tales inversiones de 
tendencia pueden explicarse por las modificaciones en la estructura poblacional con los flujos migratorios de entrada a principios de los años 2000. Prueba de ello es el gran impacto de la población migrante asentada en los centros urbanos, donde encuentran mayores oportunidades de empleo, sobre las tasas de fecundidad registradas en 2008, cuando quince Comunidades Autónomas (con la excepción de Galicia y Extremadura) ven dispararse las frecuencias de nacimientos por cada 1.000 mujeres de 15-19 años en sus respectivos territorios.

\section{COMPARACIÓN ENTRE MADRES ADOLESCENTES NACIONALES Y EXTRANJERAS}

Desde finales de los años noventa, la inmigración tiene un impacto considerable sobre la evolución de la tasa de fecundidad general en España ${ }^{7}$, también en el caso de las madres más jóvenes. Las mujeres extranjeras suelen tener hijos antes que las autóctonas (Devolder y Cabré, 2009), presentan mayores riesgos de un embarazo no planificado, independientemente de su país de origen y del nivel de estudio (Yago y Tomás, 2014) y su probabilidad de practicar una IVE es más alta en comparación con sus coetáneas españolas (Conde, 2007). Aunque la tasa de aborto adolescente baja entre 2007 y 2017, en la comparación entre españolas y extranjeras se mantiene una diferencia significativa con respecto a ese indicador: en las primeras la tasa de aborto adolescente pasa de 4,06 a 3,42 mientras que en las segundas el descenso es notable (de 22,14 a 13,99) pero los valores comparativamente son más altos.

El número de las adolescentes inmigrantes también aumenta entre 2000 y 2008, pasando del 1,9\% de la población de 10-19 años de edad al 11,8\%. En contemporáneo la proporción de los nacimientos de madres extranjeras menores de 20 años casi se

${ }^{7}$ Con la intensa llegada de población inmigrante aumenta la tasa de fecundidad de la población total de 9,2\% en 1996 a 10,7\% en 2006 y aumenta también el índice sintético de fecundidad (es un indicador del número de hijos por mujer), de 1,15 hijos a 1,33 en el mismo periodo. Asimismo, crece de forma notable la proporción de nacimientos con al menos uno de los padres extranjero: 1 nacimiento de este tipo por cada 4 nacimientos con ambos padres de nacionalidad española en 2006 mientras que en 1996 esta relación era aproximadamente de 1 por 17, produciéndose un aumento más rápido de los hijos con madre extranjera y padre español que la combinación inversa (Devolder y Cabré, 2009: 31-32). 
cuadruplica en el mismo periodo pasando de $10,7 \%$ a $38,5 \%$ (Gráfico 2).

\section{Gráfico 2 \\ Proporción de nacimientos en madres adolescentes por nacionalidad de las jóvenes (españolas y extranjeras)}

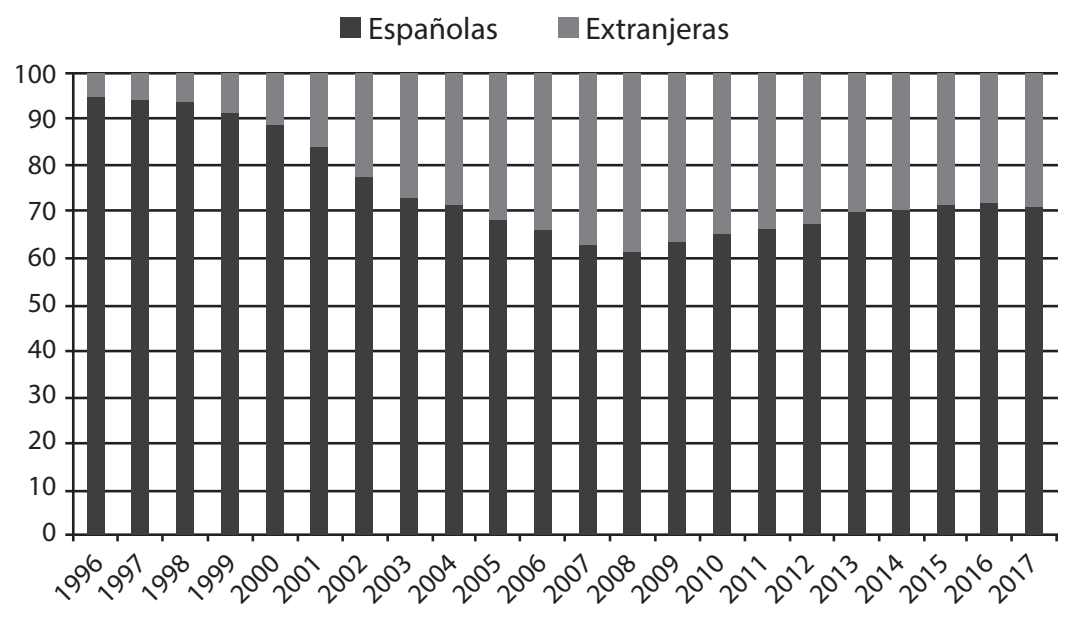

Fuente: Elaboración propia a partir de Estadística de nacimientos, Movimiento natural de la población (INE)

Tal como se evidencia en estudios anteriores (Del Campo, 1982; Yago et al., 1990: 32), las idiosincrasias que definen las prácticas afectivo-sexuales y los embarazos tempranos entre los jóvenes inmigrantes se mantienen cuando el flujo de llegada es más intenso; en un segundo momento se produce una progresiva adaptación a las características comunes del régimen de fecundidad de la sociedad receptora, una vez que se refuerza su integración, con consecuente equiparación a la proporción de los nacimientos en madres adolescentes.

Con la crisis económica de 2008 se reduce la población inmigrante en España. Pero la proporción de las madres de 1519 años extranjeras (sin incluir a las chicas nacionalizadas) sigue siendo considerable en 2017: casi seis veces más alta $(28,5 \%)$ que en 1996 (4,8\%). 
Las comparaciones entre los/las adolescentes autóctonos y los/las de otros países y residentes en España protagonizan muchos estudios sobre salud sexual, conductas de riesgos, embarazos no deseados e IVE (Conde, 2007; Bermúdez et al., 2010; Soriano-Ayala et al., 2014).

En particular, los adolescentes de origen latinoamericano y sus coetáneos españoles comparten la temprana edad de inicio en las relaciones sexuales completas (Teva et al., 2009) pero hay muchas diferencias entre estos dos colectivos en otros aspectos: los primeros tienen un mayor número de parejas sexuales que los segundos (en media 3,59 y 2,65 parejas respectivamente); en su debut sexual el $90,5 \%$ de los autóctonos utiliza algún método anticonceptivo frente el $69,6 \%$ de sus coetáneos latinos; estos últimos en menor medida ( $20 \%$ menos) utilizan anticonceptivos "casi siempre", independientemente de que sus relaciones sean ocasionales o estables, y presentan una mayor casuística de ETS.

Las jóvenes de etnia gitana siguen unas conductas sexuales similares a las que se registran entre sus coetáneas de la población inmigrante. Estudios en materia de anticoncepción señalan que en este grupo se utilizan menos los métodos anticonceptivos y presentan una mayor proporción de madres adolescentes en comparación con las no gitanas (Reig et al., 1999). Otro estudio sobre el rol de las mujeres del colectivo gitano en Andalucía (Fernando, 2009) evidencia que ellas tienen en promedio más hijos que las no gitanas y siguen pautas reproductivas diferentes: en la cultura gitana se acepta que las mujeres empiecen a tener hijos pronto y continúen teniéndolos hasta bien entrada la treintena, formando familias numerosas y redes de parentesco amplias.

\section{ESTADO CIVIL Y TIPO DE CONVIVENCIA DE LAS JÓVENES MADRES}

Las parejas juegan un papel fundamental para el bienestar y la estabilidad emotiva de la madre adolescente, pero no siempre saben (y tampoco quieren) estar a la altura de sus responsabilidades (Sánchez, 2016). Las actitudes frente a un embarazo no previsto, las decisiones con respecto a su continuidad y las formas en que se lleva psicológica y prácticamente la maternidad dependen, en 
gran medida, de la reacción y del soporte que estén dispuestos a ofrecer los padres biológicos del niño, del tipo de relación afectiva con la joven madre y de su posibilidad de compartir las tareas de crianza, aportar recursos y realizar un proyecto familiar juntos (Alberdi y Escario, 2007). Para averiguar estos aspectos nos remontamos a los datos sobre los tipos de relaciones que originan los nacimientos de esas menores de 20 años.

En la mayoría de los casos registrados por el INE entre 1996 y 2017 las adolescentes tienen su primer hijo con varones que pertenecen a la cohorte de 20-29 años (Gráfico 3), aunque los casos que presentan esta circunstancia disminuyen durante este periodo (de $63,2 \%$ a 51,4\%). La proporción de las menores de 20 años que quedan embarazadas de sus coetáneos es más reducida pero dibuja la misma trayectoria descendente, aunque sea con una intensidad inferior (pasando de $20,2 \%$ a 18,8\%). Por otra parte, crece la proporción de los padres mayores de 30 años (de $7,1 \%$ a $11,3 \%)$.

\section{Gráfico 3}

Nacimientos de madres adolescentes por grupo de edad del padre

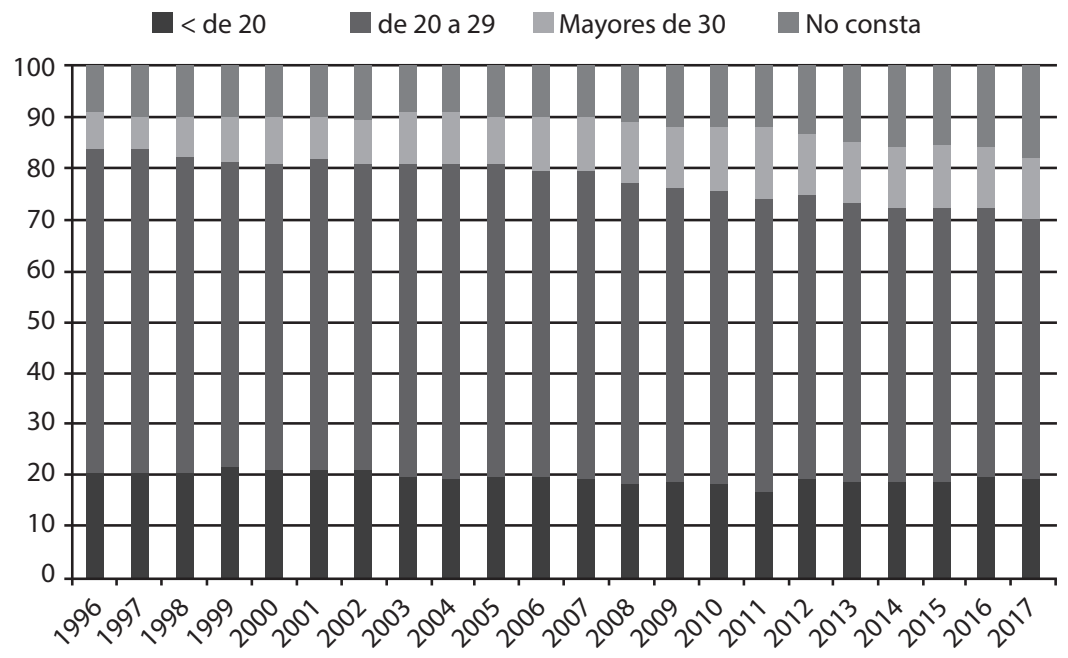

Fuente: Elaboración propia a partir de Estadística de nacimientos, Movimiento natural de la población (INE) 
Cuando las parejas son mucho más grandes de edad, la asimetría de poder en la relación es desfavorable para las jóvenes porque les resulta más difícil negociar el uso de algún anticonceptivo (en particular, el preservativo) y protegerse así contra los embarazos y las ETS (Berga, 2005). La OMS (2010) advierte que en estos casos las adolescentes corren serios riesgos de ser víctimas de sexo forzado mediante insistencias, amenazas, coacción o engaños.

Esta situación se agudiza si la adolescente es menor de los 16 años. Al respecto podemos afirmar que existen cambios legislativos que están trabajando para abordar dicha problemática. Por ejemplo con el aumento de la edad para el consentimiento sexual. España tenía una de las edades de consentimiento sexual más bajas de Europa (13 años); a partir de la reforma del Código Penal español, entrada en vigor el 1 de julio de 2015, se entiende que un/a joven posee uso pleno de razón, discernimiento y autonomía para decidir cuándo y con quién practicar sexo a partir de los 16 años.

No obstante, no siempre es posible averiguar la edad del padre biológico, el aumento de la categoría "No Consta" (de 9,6\% en 1996 a 18,3\% en 2017) con relación a los nacimientos por grupos de edad del padre es una prueba de esta circunstancia. Estos casos podrían entenderse también como un problema de registro: en el pasado no se incluía información del progenitor en las encuestas sobre nacimientos y ahora estos datos resultan todavía incompletos.

La incertidumbre sobre la identidad del padre o su ausencia hacen suponer que es frecuente la configuración de hogares monoparentales, con más dificultades de autonomía y conciliación entre vida laboral y vida familiar para las madres, a la vez que se prolonga la dependencia de sus familias de origen y/o de los sistemas asistenciales para que esté garantizado su bienestar y el de sus hijos (Ellis-Sloan, 2014).

En un contexto más conservador como la España de hace cuarenta años el embarazo en las jóvenes solía dar lugar a uniones conyugales que no estaban planificadas, pero que forzaban la constitución de hogares para dar estabilidad a las parejas con hijos en camino. No hay datos para corroborar hasta qué punto el embarazo da pié a este tipo de matrimonios "reparadores" hoy en día. Sin embargo, podemos presumir que esta tendencia se reduce por la progresiva secularización del matrimonio como "rito de paso" formal para crear una familia entre los veinteañeros españoles del siglo XXI (Alberdi, 2014). Estos acontecimientos se producen fuera 
del matrimonio, tensan las relaciones de pareja y suelen llevar a la separación cuando la llegada de un hijo no está ni deseada ni planificada por parte de ambos (Serrano, 2014).

Hasta 2015, la edad mínima para casarse era de 14 años, con consentimiento paterno o con la emancipación dictada por un juez. Con la reforma de ese año en el Código Civil, los menores que quieren casarse deben esperar hasta 16 años, coincidiendo con la edad del consentimiento sexual. Una vez que cumplen 18 años pueden casarse sin el permiso de nadie.

En la actualidad apenas hay casos de matrimonio infantil en España, después de una intensa reducción del número de bodas entre adolescentes de 15-19 años en los últimos cuarenta años. Según las Estadísticas de Matrimonio del INE, unas 55 chicas y 3 chicos menores se han casado en 2017: de ellos 40 de 17 años, 14 de 16 años, 4 de 15 años y solo 1 menor de 15; en 1975, cuando el INE empieza a recoger datos sobre este fenómeno, se casaron 6.834 menores.

Las adolescentes casadas con veinteañeros pasan de 6.402 en 1996 a 475 en 2015 y las que contraen matrimonio con hombres mayores de 30 años baja de 491 a 136.

El matrimonio temprano es habitual en el colectivo gitano, con una cultura que acepta y avala esta práctica como parte fundamental de sus costumbres ancestrales. Se trata de una institución que sirve para reforzar los lazos identitarios y de parentesco, el sentido de pertenencia y la continuidad social de este grupo étnico. El rito de la boda gitana tradicional prevé que los jóvenes novios lleguen vírgenes a ese momento y hagan muestra de su relación delante de sus familias. La unión se celebra con vista a una relación duradera y monógama, donde el reparto de los roles sociales marcan netas diferencias de género (hombre proveedor y mujer cuidadora del hogar y de la prole que engendrará). Desde los noventa los matrimonios entre adolescentes de etnia gitana son mucho menos frecuentes (San Román, 1997): si antes constituían una característica de la vida nómada de esta comunidad ahora, con el aumento del sedentarismo y una progresiva escolarización de estas jóvenes (Fernando, 2009), estos casamientos se reducen sobre todo en las áreas urbanas. Además, respecto al pasado, la condición juvenil de las mujeres gitanas dentro y fuera de su grupo étnico depende menos del logro de un proyecto familiar temprano 
y más de una formación adecuada para insertarse laboralmente (San Román, 1997).

Hasta principios de los años ochenta la maternidad extraconyugal suele asociarse con embarazos no planeados de mujeres adolescentes y jóvenes (Del Campo, 1982). Los datos del Movimiento Natural de la Población muestran que en 1980 aproximadamente 1 de cada 4 nacimientos fuera del matrimonio corresponde a mujeres menores de 20 años, frente a 1 de cada 10 nacimientos extra-conyugales en 2002 (Castro-Martín, 2007). Aunque el peso relativo de la fecundidad adolescente sea cada vez menor en el conjunto de la fecundidad no matrimonial, los nacimientos de las jóvenes madres se producen ahora fuera del matrimonio. De hecho, en 1980 solo el $13,4 \%$ de las madres adolescentes no está casadas en el momento de dar a luz, mientras que en 1996 esta proporción alcanza el $55,1 \%$ y sigue aumentando mucho hasta llegar a 89,6\% en 2015 (Gráfico 4).

\section{Gráfico 4 \\ Proporción de nacimientos en madres adolescentes por estado civil de las jóvenes (casadas y no casadas)}

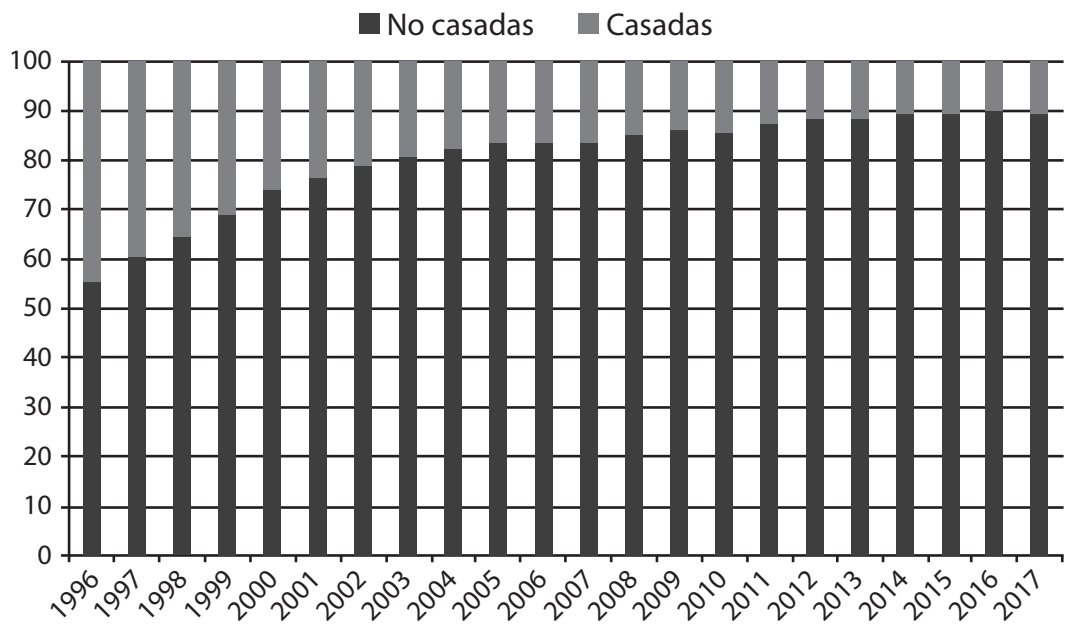

Fuente: Elaboración propia a partir de Estadística de nacimientos, Movimiento natural de la población (INE)

Nota: Entre las no casadas no se incluyen a las jóvenes madres que estaban casadas, divorciadas, viudas o separadas antes del nacimiento del hijo. 
Los nacimientos extra-conyugales aumentan en el periodo considerado. De acuerdo con la secularización y la des-tradicionalización de las relaciones de pareja en las nuevas generaciones de españoles, amor y sexualidad parecen no estar circunscritos al ámbito del matrimonio (Ayuso y García, 2014). Sin embargo, para las madres adolescentes la unión conyugal y el emparejamiento estable ayudan a la joven en la aceptación de un embarazo temprano y en plantear un proyecto familiar duradero (FPFE, 2012).

La situación es la inversa los nacimientos en uniones no estables porque es más probable que los embarazos sean no deseados, no previstos o, por lo menos en su origen, no ligados a un proyecto familiar dentro de la relación de pareja que se está conduciendo. Siguiendo esta línea interpretativa, podemos reforzar la explicación del embarazo precoz como evento ligado a la ocasionalidad de la relación afectivo-sexual mantenida, totalmente alejado, del modelo de vida en común bajo el mismo techo.

\section{UNA EMANCIPACIÓN PRECOZ Y PRECARIA}

Las madres adolescentes experimentan muchos y diversos acontecimientos vitales en un intervalo de tiempo breve e intenso, saltando etapas que las proyectan hacía unas responsabilidades adultas (Nóblega, 2009). Además de empezar su actividad sexual pronto y de tener un embarazo precoz, si consideramos también el alumbramiento de un hijo y los compromisos parentales asociados con la primera convivencia en pareja (con o sin matrimonio), la emancipación residencial, la interrupción (a veces permanente) de los estudios y la búsqueda de un trabajo para sustentarse, tendremos el cuadro completo de una adultez alcanzada de prisa, de forma no siempre planificada o esperada.

En el momento de dar a luz la mayoría de las madres adolescentes en España están solteras y viven en casa de sus padres. No hay datos detallados sobre la ocupación de las menores que forman parte de este grupo, pero podemos reseñar que en este colectivo existe una cierta disposición a ser autónomas económicamente consiguiendo un trabajo. Prueba de ello es el aumento del 5 al $10 \%$ de la proporción de las que son población activa en el mercado laboral entre 1996 y 2005, con un crecimiento ulterior de 
esta proporción en 2008 (20\%) cuando empieza la crisis económica y cuando se produce el mayor aumento de maternidades precoces en España, manteniéndose hasta $2014(21 \%)^{8}$.

En cuanto a su ocupación de las madres adolescentes casadas, el INE informa que de todos los nacimientos de la cohorte de 15-19 años ocurridos entre 2007 y 2015 de estas madres, el $57,2 \%$ realizan o comparten las tareas domésticas, otro $16,2 \%$ ocupan un puesto de trabajo cualificado, el $7,1 \%$ son trabajadoras no cualificadas y solamente el $5,4 \%$ son estudiantes.

Las dificultades relativas a la participación laboral son mayores para quienes presenta un itinerario formativo discontinuo o han interrumpido los estudios obligatorios debido al embarazo, sin conseguir retomarlos posteriormente (Berga, 2005). Esta situación es controvertida: dejar prematuramente los estudios y empezar a trabajar con vista a conseguir los recursos necesarios para la crianza es arriesgado porque las exponen a largos periodos de inestabilidad contractual, a trayectorias profesionales fragmentadas, grandes problemas para obtener puestos y sueldos de calidad, así como el riesgo de caer en la economía sumergida o transcurrir mucho tiempo en el paro (Yago y Tomás, 2014).

El embarazo y la maternidad adolescente no solamente favorecen el abandono escolar sino que son también consecuencias de itinerarios académicos negativos: se ha comprobado que el menor nivel educativo predice conductas sexuales de riesgo y de embarazos no planificados (Yago y Tomás, 2014: 404). En 2015 el $45 \%$ de las madres con una edad entre 15 y 19 ha ido a la escuela cinco o más años, sin completar las etapas de Educación General Básica, Educación Secundaria Obligatorio o Bachillerato Elemental, mientras que un $19,3 \%$ alcanza solo el graduado escolar obligatorio.

Aunque podamos encontrar a madres jóvenes que acaben con éxito estos ciclos formativos después de la llegada del hijo, el embarazo y la maternidad a edades tempranas están significativamente correlacionados con una educación apenas suficiente

${ }^{8}$ En el periodo observado las madres adolescentes casadas que son población activa no supera el $4 \%$ de todas las integrantes de esta categoría, como demostración de una mayor tendencia a depender económicamente del marido con un proyecto de vida familiar tradicional: mujeres dedicadas al cuidado doméstico y de los hijos y hombres proveedores de recursos que trabajan fuera del hogar. 
o incompleta. Se trata de una característica común para esta categoría de chicas que es posible cuantificar con precisión desde el 2008, año a partir del cual el INE inserta este dato en sus Estadísticas de Nacimientos: en ese entonces, las que llegan a dar a luz sin haber completado EGB, ESO o Bachillerato Elemental son el $51 \%$ de todas las madres adolescentes.

Para explicar este dato desde una perspectiva internacional es necesaria una aclaración: en España el embarazo adolescente se entiende como una de las causas que provocan el abandono escolar temprano, mientras que en muchos países en desarrollo el embarazo y el matrimonio precoces son consecuencias directas de un bajo nivel de escolarización (Lloyd y Young, 2009). Algunas investigaciones demuestran que el bajo nivel de escolarización ligado a la difícil adaptación al medio educativo y la existencia de problemas especiales en el aprendizaje pueden darse también antes de que se produzca el embarazo, con una mayor probabilidad de deserción y fracaso escolar durante la gestación (Yago et al., 1990: 66).

Tanto el nivel formativo alcanzado como el tipo de trabajo nos aclaran las dificultades de integración social de estas jóvenes una vez que se convierten en madres. Estos efectos negativos pueden volverse persistentes en el futuro: el acceso a la educación universitaria es prácticamente nulo para ellas mientras que las experiencias de trabajo temporal se extienden más en comparación con sus coetáneas que no han sido madres precoces (Delgado, 2011).

Existen además unas consecuencias específicas a nivel familiar y personal ligadas a este fenómeno. En primer lugar, las madres adolescentes presentan mayores probabilidades de tener más hijos en promedio una vez que llegan a la edad adulta. En segundo lugar, ellas pueden sufrir problemas a la hora de constituir un hogar estable con sus parejas con mayor frecuencia en comparación con las que han tenido hijos después de haber cumplido 25 años (Samuels et al., 1994). Finalmente, estas jóvenes pueden transmitir su vulnerabilidad social a la prole (Wilkinson y Pickett, 2009), con el riesgo de que sus hijos reproduzcan los mismos historiales formativos precarios y las mismas conductas afectivosexuales de riesgo que sus madres cuando sean adolescentes (Duncan, 2007). 


\section{CONCLUSIONES}

La maternidad es una de las transiciones vitales más trascendentales para las mujeres. Sin embargo, cuando ocurre en edad adolescente se entiende como un acontecimiento prematuro, inoportuno e indeseable que altera (o debilita) los itinerarios normalizados de desarrollo personal e inserción social de la joven (Nóblega, 2009), principalmente por su falta de autonomía e independencia o por su incapacidad para asumir el embarazo y la crianza de un niño en unas condiciones físicas, psicológicas, sociales y económicas idóneas (Berga, 2005).

Hoy en día los nacimientos de madres adolescentes en España son mucho menos respecto a los años setenta del siglo pasado, pero en los últimos años el fenómeno ha alcanzado una cierta trascendencia pública y en el debate social y político del país sobre la salud sexual y reproductiva, las relaciones de género y de pareja y los modelos de planificación familiar y transición a la vida adulta de las nuevas generaciones.

El repaso estadístico realizado en este artículo ayuda a entender los embarazos y la maternidad en edad adolescente como problemas urgentes de salud pública. Por ello, esperamos que desde la arena política se siga apostando por una educación preventiva y exhaustiva en salud sexual para todos los/las adolescentes, además de una atención socio-sanitaria acorde con las circunstancias personales, familiares y sociales de cada madre menor de edad desde los centros escolares y desde los servicios de asistencia socio-sanitaria.

\section{BIBLIOGRAFÍA}

Alberdi, I. y Escario, P. (2007). Los hombres jóvenes y la paternidad, Madrid, Fundación BBVA.

Alberdi, I. (2014). "El retraso de la procreación y el mantenimiento de la juventud", Metamorfosis, 0, 9-25.

Ayuso Sánchez, L. y García Faroldi, L. (2014). Los españoles y la sexualidad en el Siglo XXI, Madrid, Centro de Investigaciones Sociológicas.

Berga Timoneda, A. (2005). "Adolescencia femenina y riesgo social: una relación invisible”, Educación Social, 29, 63-78. 
Bermúdez, M.; Castro, Á.; Madrid, J.; Buela-Casal, G. (2010). "Análisis de la conducta sexual de adolescentes autóctonos e inmigrantes latinoamericanos en España", International Journal of Clinical and Health Psychology, 10(1), 89-103.

Castro-Martín, T. (2007). "Maternidad sin matrimonio. Nueva vía de formación de familias en España", Documentos de Trabajo n.16, Madrid, Fundación BBVA.

Conde, F. (2007). La interrupción voluntaria del embarazo en población adolescente en el sector inmigrante, recuperado en http://www.msc.es/organizacion/sns/planCalidadSNS/pdf/ equidad/IVES_Monografico_Juventud_Inmigrante.pdf

Del Campo, S. (1982). La evolución de la familia española en el siglo XX, Madrid, Alianza.

Delgado, M. (2011). Pautas anticonceptivas y maternidad adolescente en España, Madrid, CSIC y Sociedad Española de Contracepción.

Devolder, D. y Cabré, A. (2009). "Factores de la evolución de la fecundidad en España en los últimos 30 años", Panorama Social, 10, 23-39.

Díaz-Aguado, M.J. (2006). "Sexismo, violencia de género y acoso escolar. Propuestas para una prevención integral de la violencia", Revista de Estudios de Juventud, 73, 38-58.

Duncan, S. (2007). "What's the problem with teenage parents? And what's the problem with policy?", Critical Social Policy, 27(3), 307-334.

Ellis-Sloan, K. (2014). "Teenage Mothers, Stigma and their Presentations of Self"', Sociological Research Online, 19(1), 1-13.

Fernando Serrano, L. (2009). "Mujeres gitanas", ponencia presentada en el I Congreso Virtual sobre Historia de las Mujeres, 15-31 de octubre de 2009, Jaén.

FPFE (Federación de Planificación Familiar Estatal). (2012). Factores que influyen en el embarazo de adolescentes y jóvenes. Aprendiendo de las experiencias de las jóvenes, Proyecto Europeo "Sexual Awareness Europe" (SAFE-II), Madrid.

Grupo Daphne. (2009). III Encuesta Bayer Schering Pharma sobre Sexualidad y Anticoncepción en la Juventud española, Madrid. 
Grupo Daphne. (2011). VII Encuesta de Anticoncepción en España, Madrid.

INJUVE (Instituto de la Juventud). (2008). Jóvenes, salud y sexualidad, Estudio IJ132, Madrid, Ministerio de Sanidad, Servicios Sociales e Igualdad.

INJUVE (Instituto de la Juventud). (2010). Sondeo de opinión: jóvenes y diversidad sexual. Madrid, Ministerio de Sanidad, Servicios Sociales e Igualdad.

INJUVE (Instituto de la Juventud). (2012). Informe Juventud en España 2012. Cifras jóvenes, Madrid, Ministerio de Sanidad, Servicios Sociales e Igualdad.

Lailla, J. (2011). "La sexualidad en el adolescente en España comparada con otros estados europeos", en García-Tornel, S.; Miret, P.; Cabré, A.; Flaquer, L.; Berg-Kelly; K., Roca G.; Elzo, J.; Lailla, J. (coords.) El adolescente y su entorno en el siglo XXI. Instantánea de una década. Esplugues de Llobregat: Observatorio de salud de la infancia y la adolescencia, 121-136.

Lloyd, C. y Young, J. (2009). New Lessons: The Power of Educating Adolescent Girls, Nueva York, Population Council.

Madrid, J. (2009). "El preservativo entre los más jóvenes", Evidencias en Pediatría, 5(34).

Megías, I.; Rodríguez, E.; Méndez, S. y Pallarés, J. (2005). Jóvenes y sexo. El estereotipo que obliga y el rito que identifica, Madrid, Fundación de Ayuda contra la Drogadicción, Instituto de la Juventud y Caja Madrid.

Ministerio de Sanidad y Consumo. (2007). La interrupción voluntaria del embarazo y los métodos anticonceptivos en jóvenes, Madrid, Ministerio de Sanidad y Consumo, Centro de Publicaciones Oficiales.

Nóblega, M. (2009). "La maternidad en la vida de las adolescentes: implicancias para la acción”, Revista de Psicología, 28(1), 29-54.

OMS (Organización Mundial de la Salud). (2010). Salud de los adolescentes. Disponible en www.who.int/topics/adolescent_ health/es/

OMS (Organización Mundial de la Salud). (2014), Adolescent Pregnancy. Fact Sheet 14.08. Disponible en http://apps.who. int/iris/bitstream/10665/112320/1/WHO_RHR_14.08_eng.pdf 
Reig S., Curos S., Balcells J., Batalla C., Ezpeleta A., Comin E. (1999). "Anticoncepción: gitanas frente a payas", Atención Primaria, 23, 63-67.

San Román, T. (1997). La diferencia inquietante: viejas y nuevas estrategias culturales de los gitanos, Madrid, Siglo XXI.

Sánchez Basilio, C. (2016). "Percepciones de la paternidad en los varones adolescentes de la Comunidad Autónoma de Madrid", Nure Investigación, 13(80).

Save the Children. (2012). Every woman's right. How family planning saves children's lives. Londres, Save the Children Fund.

SEC (Sociedad Española de Contracepción). (2014). Encuesta Anticoncepción SEC 2014, Madrid, SEC.

SEC (Sociedad Española de Contracepción). (2018). Encuesta Anticoncepción SEC 2018, Madrid, SEC.

Serrano Fuster, I. (2014). Sexo con sentido. Para jóvenes, Madrid, Síntesis.

Soriano-Ayala, E.; González-Jiménez, A.; Soriano-Ferrer, M. (2014). "Educación para la salud sexual. Del enamoramiento al aborto. Un estudio cualitativo con adolescentes españoles e inmigrantes", Perfiles Educativos, XXXVI(144), 105-119.

Teva, I.; Bermúdez, M.; Buela-Casal, G. (2009). "Conductas de riesgo para la infección por el $\mathrm{VIH}$ y las enfermedades de transmisión sexual (ETS) en adolescentes en el año 2007: diferencias en función de variables socio-demográficas", Revista Española de Salud Pública, 83, 309-320.

Yago Simón, T.; Zapatero, S.; Palacios del Campo, J.; Pastor, M.P.; Jimeno, A. (1990). Maternidad Juvenil, Zaragoza, Área de Sanidad y Acción Social del Ayuntamiento de Zaragoza.

Yago Simón, T. y Tomás Aznar, C. (2014). "Variables socio-demográficas relacionadas con embarazos no planificados en jóvenes de 13 a 24 años", Revista Española de Salud Pública, 88, 395-406.

Wilkinson, R. y Pickett, K. (2009). Desigualdad: un análisis de la (in)felicidad colectiva, Barcelona, Noema. 